\title{
VERO cells harbor a poly-ADP-ribose belt partnering their epithelial adhesion belt
}

Poly-ADP-ribose (PAR) is a polymer of up to 400 ADP-ribose units synthesized by poly-ADPribose-polymerases (PARPs) and degraded by poly-ADP-ribose-glycohydrolase (PARG). Nuclear PAR modulates chromatin compaction, affecting nuclear functions (gene expression, DNA repair). Diverse defined PARP cytoplasmic allocation patterns contrast with the yet still imprecise PAR distribution and still unclear functions. Based on previous evidence from other models, we hypothesized that PAR could be present in epithelial cells where cadherin-based adherens junctions are linked with the actin cytoskeleton (constituting the adhesion belt). In the present work, we have examined through immunofluorescence and confocal microscopy, the subcellular localization of PAR in an epithelial monkey kidney cell line (VERO). PAR was distinguished colocalizing with actin and vinculin in the epithelial belt, a location that has not been previously reported. Actin filaments disruption with cytochalasin $D$ was paralleled by PAR belt disruption. Conversely, PARP inhibitors 3-aminobenzamide, PJ34 or XAV 939, affected PAR belt synthesis, actin distribution, cell shape and adhesion. Extracellular calcium chelation displayed similar effects. Our results demonstrate the existence of PAR in a novel subcellular localization. An initial interpretation of all the available evidence points towards TNKS-1 as the most probable PAR belt architect, although TNKS-2 involvement cannot be discarded . Forthcoming research will test this hypothesis as well as explore the existence of the PAR belt in other epithelial cells and deepen into its functional implications. 
1 VERO cells harbor a poly-ADP-ribose belt partnering their epithelial adhesion belt

2 Laura Lafon-Hughes ${ }^{1}$, Salomé C. Vilchez Larrea ${ }^{2}$, Alejandra Kun ${ }^{3,4}$, Silvia H. Fernández

3 Villamil ${ }^{2,5}$

$4{ }^{1}$ Departamento de Genética, Instituto de Investigaciones Biológicas Clemente Estable (IIBCE),

5 Montevideo, Uruguay; ${ }^{2}$ Instituto de Investigaciones en Ingeniería Genética y Biología Molecular

6 “Dr. Héctor N. Torres”, Consejo Nacional de Investigaciones Científicas y Técnicas, Ciudad

7 Autónoma de Buenos Aires, Argentina; ${ }^{3}$ Departamento de Proteínas y Ácidos Nucleicos, Instituto

8 de Investigaciones Biológicas Clemente Estable (IIBCE), Montevideo, Uruguay; ${ }^{4}$ Departamento

9 de Biología Celular y Molecular, Sección Bioquímica, Facultad de Ciencias, Universidad de la

10 República, Montevideo, Uruguay; ${ }^{5}$ Departamento de Química Biológica, Facultad de Farmacia y

11 Bioquímica, Universidad de Buenos Aires, Ciudad Autónoma de Buenos Aires, Argentina

12 Corresponding authors:

13 Laura Lafon- Hughes; lauralafon2010@gmail.com; IIBCE: Avenida Italia 3318, +598 2487

$141616,135$.

15 Silvia Fernández Villamil; s.villamil@ingebi.conicet.gov.ar, INGEBI-CONICET. Vuelta de

16 Obligado 2490, Ciudad Autónoma de Buenos Aires (1428), Buenos Aires, Argentina. + 5411

$17 \lcm{47832871}$

18 RUNNING TITLE: Poly-ADP-ribose in VERO epithelial belt 


\section{ABSTRACT}

20 Poly-ADP-ribose (PAR) is a polymer of up to 400 ADP-ribose units synthesized by poly-ADP-

21 ribose-polymerases (PARPs) and degraded by poly-ADP-ribose-glycohydrolase (PARG). Nuclear

22 PAR modulates chromatin compaction, affecting nuclear functions (gene expression, DNA

23 repair). Diverse defined PARP cytoplasmic allocation patterns contrast with the yet still imprecise

24 PAR distribution and still unclear functions. Based on previous evidence from other models, we

25 hypothesized that PAR could be present in epithelial cells where cadherin-based adherens

26 junctions are linked with the actin cytoskeleton (constituting the adhesion belt). In the present

27 work, we have examined through immunofluorescence and confocal microscopy, the subcellular

28 localization of PAR in an epithelial monkey kidney cell line (VERO). PAR was distinguished

29 colocalizing with actin and vinculin in the epithelial belt, a location that has not been previously

30 reported. Actin filaments disruption with cytochalasin D was paralleled by PAR belt disruption.

31 Conversely, PARP inhibitors 3-aminobenzamide, PJ34 or XAV 939, affected PAR belt synthesis,

32 actin distribution, cell shape and adhesion. Extracellular calcium chelation displayed similar

33 effects. Our results demonstrate the existence of PAR in a novel subcellular localization. An

34 initial interpretation of all the available evidence points towards TNKS-1 as the most probable

35 PAR belt architect, although TNKS-2 involvement cannot be discarded . Forthcoming research

36 will test this hypothesis as well as explore the existence of the PAR belt in other epithelial cells

37 and deepen into its functional implications. 


\section{Introduction}

Poly-ADP-ribose (PAR) is a linear or branched polymer of up to 400 ADP-ribose units that binds (covalently or not) to target proteins. PAR is synthesized by poly-ADP-ribose-polymerases (PARPs) and the key catabolic enzyme is poly-ADP-ribose-glycohydrolase (PARG). PAR synthesis involves the cleavage of $\mathrm{NAD}^{+}$into ADP-ribose (monomers) and free nicotinamide (Virag and Szabo 2002). A steady-state balance is maintained in normal cells regarding PAR synthesis and degradation. An excellent review on the roots and developments of PARylation research has been published recently (Virag 2013).

As alterations in PARP or poly-ADP-ribosylation (PARylation) levels are detected in several pathological conditions (Cerboni et al. 2010; Masutani et al. 2005; Strosznajder et al. 2012; Virag and Szabo 2002), and PARP or PARG inhibition interferes with T. cruzi infection and proliferation of the parasite (Vilchez Larrea et al. 2012; Vilchez Larrea et al. 2013), PAR biology studies may have far reaching biomedical implications.

PARP gene family includes catalytically inactive members (i.e. ARTD-9 and-13), several members with just mono(ADP-ribosyl)ating (MARylating) activity from which only one has been mapped to submembrane domains (ARTD8 in focal adhesions) and members with putative (tankyrase-2) or proved enzymatic PARylating activity (Hassa and Hottiger 2008; Hottiger et al. 2010; Vyas et al. 2013). A different gene family codes membrane-bound or secreted MAR-(or even PAR)-synthesizing enzymes, whose activity is always extracellular: ecto- ADP-ribosyltransferases (ARTC-1 to 5) (Morrison et al. 2006, Hottiger et al 2010).

Interestingly, different PARPs may have different PARylating activities. For example, tankyrase-1 (TNKS-1) synthesizes oligomers of an average chain length of 20 units without detectable branching while PARP-1 synthesizes large linear or branched polymers (Hottiger et al. 2010).

A plethora of PARP inhibitors can be used to envisage the involvement of different members of PARP family in specific processes. These compounds display diverse binding and in vitro potencies towards PARP-1 and TNKS-1 (Table 1)Human PARG is expressed in alternative splice variants yielding isoforms that localize to different cell compartments. (Bonicalzi et al. 2005; Bonicalzi et al. 2003; Ohashi et al. 2003). Cytoplasmic PARG accounts for most of the PARG activity in cells (Meyer-Ficca et al. 2004). Although most PARG activity would be cytoplasmic and most PARP family members can be detected in the cytoplasm, their role inside the nucleus 
68

69

70

71

72

73

74

75

76

77

78

79

80

81

82

83

84

85

91

92

93

has been better studied. PARP-1 (the single family member located exclusively in the nucleus), nuclear PARP-2 and -3 compete with histone deacetylases for NAD ${ }^{+}$consumption. Poly-ADPribosylation of chromatin-associated proteins usually correlates with increased histone acetylation, decreased DNA methylation and low chromatin compaction. Thus, PARylation may modulate gene expression and facilitate the access of DNA repair machinery to damaged sites (Tulin and Spradling 2003). In fact, PARP-1, the most conserved and best studied PARP, plays a role in the recognition of DNA damage. Nevertheless, PARylation has also been reported in heterochromatic contexts (i.e. X chromosome inactivation) (Burkle and Virag 2013; Dantzer and Santoro 2013; Lafon-Hughes et al. 2008).

TNKS-1 maps to endoplasmic reticulum, Golgi, secretion vesicles, epithelial lateral membrane or lysosomes (Bottone et al. 2012; Chi and Lodish 2000; Hsiao and Smith 2008; Vyas et al. 2013; Yeh et al. 2006). TNKS-1 can also be recruited to the nucleus by TRF1 (telomere repeat binding factor 1) and accompany NuMa (Nuclear/ Mitotic apparatus protein) in spindle poles (Hsiao and Smith 2008). In MDCK (renal epithelial) cells, TNKS-1 is recruited from the cytoplasm to the lateral plasma membrane upon formation of E-cadherin-based cell-cell contacts (Yeh et al. 2006). Extracellular calcium chelation, which prevents cell-cell adhesion, displaces TNKS-1 (Yeh et al. 2006). E-cadherin binds alpha- catenin and vinculin, actin-binding proteins present at the adherence junctions linking actin microfilaments to cadherin. As vinculin and catenin have been recovered as PARylated proteins in co-immunoprecipitation experiments (Gagne et al. 2008; Gagne et al. 2012), we hypothesized that PAR (synthesized by TNKS-1) would be detectable associated to the adherens junctions. It is envisaged that PAR abundance or scarcity could affect the epithelial structure as well as transcendent critical cell signaling pathways, particularly in pathological situations.

In the present work, we have described through immunofluorescence and confocal microscopy, the subcellular localization of PAR in an epithelial monkey kidney cell line (VERO). In fact, we detected PAR associated to the epithelial belt, in a location that has not been previously reported. We have used PARP inhibitors to demonstrate that the immunodetected signal associated to the epithelial belt is PAR and that if PAR synthesis is precluded, actin cytoskeleton as well as cell shape and cell adhesion are affected. Based on these data and previously reported information, TNKS-1 poses as a plausible candidate as the PARP responsible for PAR synthesis in the epithelial belt. However, the participation of other PARPs, particularly TNKS-2, cannot be ruled out. TNKS-1 knockdowns have proven to be unviable since mitosis and cell viability are 
100 dramatically affected (Vyas et al. 2013). Therefore, the mechanisms involved in PAR belt 101 formation will be studied in the future by the implementation of other approaches. 
102 Materials and methods

103 Cell culture

104 Cercopithecus aethiops (green monkey) VERO cells (ATCC CCL-81 (Faral-Tello et al. 2012)

105 were cultured in MEM (PAA E15-888) supplemented with 10 percent FBS (PAA A15-151) and 2

$106 \mathrm{mM} \mathrm{L-glutamine}$ at $37^{\circ} \mathrm{C}$ and 5percent $\mathrm{CO}_{2}$. To perform the experiments, cells were seeded in

107 complete media in 24-well plates on $12 \mathrm{~mm}$-diameter coverslips.

108 Treatments were continuous and carried in duplicates, in parallel with a common (duplicate)

109 control and the correspondent controls without primary antibodies.

110 Cytoskeleton disruption

111 Cytochalasin D (GIBCO PHZ 1063; 2 microM and 20 microM) was added 30 min before

112 fixation.

113 Incubation with PARP inhibitors or a calcium chelator

114 Cells were incubated with PAR synthesis inhibitors, namely $5 \mathrm{mM}$ 3-aminobenzamide (3-AB, 115 SIGMA A-0788), 80 microM PJ34 (CALBIOCHEM 528150) or 25 microM XAV 939 (abcam

116 120897), concomitant to seeding. Extracellular calcium deprivation with $3 \mathrm{mM}$ EGTA was also

117 assayed following the same experimental schedule. In all these cases, cells were fixed $5 \mathrm{~h}$ after

118 the continuous treatment initiation. Alternatively, the established monolayers were exposed to

119 different PARP inhibitors treatments: 5mM 3AB (24 h), $80 \mu \mathrm{M}$ PJ34 (1 h, 5 h, 7 h), $25 \mu \mathrm{M}$ XAV

120939 (12 h, 24 h) or 250 nM Olaparib (JS Research Chemicals Trading; 6 days) and then were

121 subject to fixation.

122 Immunostaining

123 Cells were washed in filtered PBS (fPBS, 0.22 microm pore size), fixed in 4 percent

124 paraformaldehyde (PFA, unless otherwise stated) in fPBS $15 \mathrm{~min}$ at $4^{\circ} \mathrm{C}$ or in 10 percent

125 trichloroacetic acid (TCA; see supplementary Fig. S1), washed in fPBS, permeabilized in

1260.1 percent Triton-X100 in fPBS, and immersed in blocking buffer $(0.2$ percent Tween, 1 percent

127 BSA in fPBS) for $30 \mathrm{~min}$. An indirect immunostaining procedure was performed. Briefly, cells

128 were incubated with the specific antibodies, namely 1:1500 rabbit polyclonal anti-PAR (Beckton

129 Dickinson BD551813), 1:1000 Tulip chicken polyclonal anti-PAR (\#1023), 1:1000 or 1:100 H10

130 clone mouse monoclonal anti-PAR antibody (Tulip \#1020), or 1:100 mouse anti-vinculin (abcam

131 18058) diluted in blocking buffer for $2 \mathrm{~h}$ at $37^{\circ} \mathrm{C}$. After washing in fPBS/T (0.1percent Tween), 
132 sections were incubated ( $1 \mathrm{~h}, \mathrm{RT})$, with the correspondent anti-antibodies mix (1:500 to 1:250

133 anti-mouse-Cy3, 1:1000 anti-rabbit-Alexa 488, 1:500 goat anti-chicken DyLight 488) in blocking

134 buffer for $1 \mathrm{~h}$ at RT. When pertinent, 1: 150 phalloidin (Molecular Probes R415 or A22283) was

135 included in the mix. After washing in fPBS/T and fPBS, DAPI counterstaining ( $1.5 \mathrm{microg} / \mathrm{mL}$ in

136 fPBS) and a final wash in fPBS, coverslips were mounted in Vectashield (Vector 94010) and

137 sealed with nail polish. Controls without primary antibody were run in parallel to check the

138 specificity of the detected signals. Besides, a control avoiding the permeabilization step was done

139 in order to check if PAR signal was due to the presence of intracellular or extracellular polymer.

140 Confocal microscopy and image analysis

141 Single images or image stacks were recorded with an Olympus FV300 with a Plan Apo 60x/1.42

142 NA oil immersion objective or a Leica TCS SP5 II confocal microscope with a Plan Apo 63x/1.4

143 NA (or a Plan Apo 100x/1.4 NA) oil immersion objective, with or without digital zoom. To

144 assure signal specificity, original images were taken in the same conditions as reference images

145 of cells not labeled with primary antibodies, at the same confocal session. ImageJ free software

146 was used for image processing (including brightness/contrast adjustment and Gaussian blur

147 filtering). 
149 Untreated Vero cells harbor different nuclear and peripheral PAR polymers

150 Poly-ADP-ribose was detected in nuclear and peripheral localizations, using the BD anti-PAR 151 antibody. These signals were detected after 10 percent TCA or 4 percent PFA fixation. Given that

152 TCA causes protein precipitation, a stronger background was detected in the absence of primary 153 antibody; therefore, PFA was selected for subsequent experiments (Fig. S1).

154 Since it has recently been demonstrated that at least one member of the ecto-ARTC family can

155 catalyze the synthesis of short lineal PAR chains on the extracellular side of the plasma

156 membrane (Morrison et al. 2006), we decided to check whether the detected epitope was

157 intracellular or extracellular. Hence, immunolocalization was performed avoiding the

158 permeabilization step (in parallel to the routine protocol). In the absence of permeabilization,

159 neither the nuclear nor the peripheral PAR signals were detected (Fig. S2), demonstrating the

160 intracellular nature of the epitope.

161 Immunostaining with different primary antibodies in parallel yielded apparently conflicting 162 results. For example, nuclear PAR was detected with polyclonal rabbit BD or chicken Tulip anti163 PAR antibodies (Fig. 1), but not with Tulip monoclonal H10 clone antibody. According to the 164 respective data sheets, the three antibodies were generated against PAR of unknown length and 165 branching conjugated to methylated BSA. Nevertheless, the latter antibody has known specificity 166 for long PAR chains (above 20 residues; Kawamitsu et al. 1984) and has been widely used to 167 monitor the nuclear response to DNA damage, which is mainly PARP-1 dependent (Vodenicharov 168 et al. 2005, Gagné et al. 2008). PARP-1 synthesizes long branched chains (Hottiger et al. 2010). 169 Coherently, DNA damage response proteins such as p53 or XPA form complexes mainly in the 170 presence of long PAR chains (Fahrer et al. 2007). In fact, while short PAR chains (16-mer) do not 171 interact with XPA and form a single complex with p53, long PAR chains (55-mer) promote the 172 formation of a complex with XPA and three specific complexes with p53 (Fahrer et al. 2007).

173 PAR belt was detected with BD rabbit anti-PAR antibody (\#551813) but not with Tulip chicken 174 anti-PAR antibody (\#1023) (Fig. 1), suggesting again the existence of a differential structure of 175 both PAR polymers. Interestingly, this is not the first report of differential recognition of PAR 176 polymers by antibodies. For example, 16B antibody, which has a preference for branching 
177 regions, recognizes just 50 percent of PAR polymer detectable by H10 (Kawamitsu et al. 1984).

178 Although this phenomenon is more likely to occur with monoclonal antibodies, it seems to be

179 also true for some polyclonal antibodies. In any case, this PAR would correspond to short-chain

180 polymer (up to 20-mer), not recognizable by H10, as expected under the hypothesis that belt PAR

181 is an oligomer (up to 20 units) synthesized by TNKS-1.

182 Specific PARP inhibition alters microfilament distribution

183 In VERO cells, PARP-1 is exclusively nuclear (data not shown). The nuclear PARPs inhibitor 184 OLA (250 nM, 6 days; Narwal et al. 2012) depleted nuclear PAR without affecting belt PAR (Fig $185 \mathrm{~S} 3$ ). Treatments with other PARP inhibitors (5mM 3AB for $24 \mathrm{~h}, 80 \mu \mathrm{M}$ PJ34 up to $7 \mathrm{~h}, 25 \mu \mathrm{M}$ 186 XAV 939 up to $24 \mathrm{~h}$ ) applied on the established monolayer had no effect on the PAR belt (data 187 not shown). Taken together, these results suggest that belt PAR is characterized by extremely high 188 stability.

189 To confirm PAR identity, we evaluated the influence of PARP inhibitors (5 mM 3-AB or 80 190 microM PJ34) on cellular PAR synthesis. To that end, inhibitors were added at the moment of cell 191 seeding (Fig. 2, green). 3-AB slightly affected PAR while PJ34 showed a stronger effect, 192 particularly on peripheral PAR. The combined treatment yielded a result similar to PJ34 alone. 3$193 \mathrm{AB}$ is a non potent PARP inhibitor with detectable binding to PARP-1 but undetectable effect on 194 TNKS-1 (Whalberg et al. 2012, Riffell et al. 2012, Table 1). PJ34 binds both nuclear PARPs and 195 TNKS with higher affinity than 3-AB, but the IC50 for PARP-1 is nearly 30 times lower than that 196 reported for TNKS-1. Thus, it affects PARP-1 activity preferentially, but inhibits TNKS-1 and-2 197 in a non-negligible proportion.

198 In 3-AB and PJ34 experiments, rhodamine- phalloidin (Fig. 2, red) allowed the concomitant 199 detection of the actin cytoskeleton.

200 3-AB displayed a border-line effect on PAR and actin distribution (Fig. 2 C, H, M). PJ34 induced 201 a deeper blocking effect on PAR belt synthesis (Fig. 2 D, I, N) and the strongest effect was 202 obtained with the combined drugs (Fig. 2 E, J, O). Notice that in spite of diminished belt 203 synthesis, there has been no diminution in cellular density. However, cell shape appeared semi204 rounded in some cases and cell area is diminished, reflecting incomplete spread out of the cells. 205 The vehicle, DMSO, affected neither PAR nor the actin belt (Fig. 2 B, G, L). 
206 To sum up, PARP inhibitors affected not only the PAR belt but also the distribution of actin

207 filaments, suggesting the existence of a physical direct or indirect interaction of PAR with the 208 actin cytoskeleton.

209 Peripheral PAR colocalized with cortical actin and vinculin in the epithelial belt

210 VERO epithelial cells present adhesion belts separating apical and basal domains, with cortical 211 actin filaments anchored to the belts. To analyze PAR localization in more detail, confocal stacks 212 of cells immunostained for PAR and co-stained with phalloidin (Fig. 3) or co-immunostained to 213 detect vinculin (Fig. 4), were used. Figure 3 highlights the fact that PAR is associated to sub 214 membrane domains only in the proximity of a neighbor cell. PAR distribution in the intercellular 215 limits, novel to our knowledge, showed a well defined pattern consisting of two parallel 216 punctuated lines in intercellular not fully formed contact regions (Fig. 3 D-F double arrows) and 217 present as a single punctuated line in completely joined cells (Fig. $3 \mathrm{C}$ single arrows) but absent 218 in membrane/cortical domains without neighbor cells (Fig. 3 arrowheads). PAR was located at 219 the place where cortical filaments were anchored, as evidenced by the unequal filament direction 220 between both sides of the intercellular limit/adhesion belt. PAR seemed to be a partner of cortical 221 actin filaments. Z-stacks revealed the existence of a structure that we called the "PAR belt", with 222 a height of around 1 to 1.5 microm (up to 4 slices every 0.5 microm). The clear-cut presence of 223 PAR in intercellular junctions (arrows) but not associated to the plasma membrane in neighbor224 free domains (arrowheads) is illustrated in Fig. 3 orthogonal views (G-R). The yellow lines 225 indicate the cutting planes.

226 Vinculin is an actin-binding protein that displays a dual localization: basal and apical, related to 227 cell-matrix focal adhesions and to ZO-1 positive tight junctions in the epithelial belt, respectively 228 (Maddugoda et al. 2007). Interestingly, while focal adhesion vinculin is not PARylated (Fig. 4, 229 arrowheads), a colocalization of PAR (in green) and vinculin (in red) is observed at the apical 230 position correspondent to the epithelial belt (Fig. 4, arrows).

231 During actin cytoskeleton disruption, PAR accompanied actin

232 In order to test the physical association of PAR to the actin cytoskeleton in this particular 233 localization, we induced microfilaments disassembly through cytochalasin D (2 and 20 microM, $23430 \mathrm{~min}$ ) treatment. While in control cells the actin/ PAR belt could clearly be observed (Figure 5 235 A-D, arrow), after 30 min of low-dose cytochalasin D treatment $(2 \mu \mathrm{M})$ the actin belt was 
236 fractured. In some cells, belt microfilaments could still be observed (Figure $5 \mathrm{E}-\mathrm{H}$, arrows),

237 although they looked non-continuous and tensionless; in other cells, the actin/ PAR belt had

238 disappeared (Figure $5 \mathrm{E}-\mathrm{H}$, arrowheads). After exposure to a stronger treatment (20 $\mu \mathrm{M}$

239 cytochalasin D), no filamentous structure remained. Instead, there was a punctuated actin pattern

240 with some precipitates (Fig. 5 I-L, arrow) colocalizing with PAR. Therefore, belt PAR

241 accompanied actin microfilaments during their structural loss.

242 EGTA or XAV939 disturbed PAR belt synthesis, affecting the actin cytoskeleton, cell shape and 243 cell adhesion

244 We reasoned that in a condition in which TNKS-1 was not recruited to the epithelial belt, 245 peripheral PAR would not be synthesized. It is well established that extracellular $\mathrm{Ca}^{2+}$ chelation 246 hampers cell adhesion. More recently, it has been shown that TNKS-1 is recruited from the 247 cytoplasm to the lateral plasma membrane upon formation of E-cadherin-based cell-cell contacts 248 in renal epithelial cells, and the recruitment depends on extracellular calcium ion (Yeh et al. 249 2006). Thus, we depleted extracellular calcium with EGTA (3 mM). Under this condition, cell 250 roundness and diminished cell adhesion leading to reduced and irregular cell density were 251 observed (although not reflected in the photographs because empty fields were not 252 photographed). Concomitantly with cell roundedness, PAR diminution was observed (Fig. 6 B, E, $253 \mathrm{H}$ ), as expected under our hypothesis.

254 EGTA chelation is a very unspecific treatment. Thus, we next exposed cells since the moment of 255 seeding to XAV 939, an inhibitor which exhibits a strong preference for TNKSs over other 256 PARPs (Table 1; Wahlberg et al. 2012; Riffell et al. 2012). Like with EGTA, a decrease in cell 257 density was repeatedly observed (Fig. 6 C, F, I). As the time interval was short (just 5 h), this 258 cannot be explained by a reduction in the number of cell cycles, but by diminished cell 259 attachment. This effect (Fig. 6 C, F, I) is stronger than the one observed with PJ34 + 3AB (Fig. 2 260 E, J, O) which had reduced cell spreading area without lessening the number of monolayer261 constituting cells. There were a plethora of cell shapes including round and binucleated cells. 262 Finally, while in control populations it was difficult to find an isolated cell pair (a confluent 263 monolayer was almost everywhere), in XAV-treated populations cell pairs were frequent, but 264 many times the PAR belt junction was incomplete. To sum up, XAV 939 displayed a strong effect 
265 on the cell junction regions, with diminished cell attachment, increased roundness and partial loss 266 of PAR/actin belt.

\section{Conclusions}

268 In the present work we have shown for the first time the existence of a PAR belt associated to the 269 actin cytoskeleton and colocalizing with the anchorage protein vinculin. Vinculin associates to 270 the E-cadherin complex. Thus, it is expected that PAR interacts with several members of the 271 complex. Although our data fits the reported vinculin/alpha-catenin co-immunoprecipitation with 272 anti-PAR antibodies (Gagne et al. 2008; Gagne et al. 2012), in our system, given the resolution of 273 confocal microscopy, we did demonstrate that the cell junction apparatus (not necessarily nor 274 exclusively vinculin) is PARylated.

275 Actin cytoskeleton disruption affects the PAR belt whereas the interference with PAR belt 276 synthesis leads to actin cytoskeleton, cell shape and cell adhesion changes.

277 The graded effects obtained with 3AB, PJ34 and XAV 939 indicate a probable involvement of 278 TNKSs in PAR belt synthesis. We cannot formally exclude TNKS-2 as the responsible enzyme.

279 Parsimony favors the hypothesis of TNKS-1 involvement in PAR belt synthesis, since: (1) In 280 VERO cells, belt PAR is not detected with an antibody targeting long PAR chains (TNKS-1 281 synthesizes short chains); (2) in another renal epithelial cell line (MDCK), TNKS-1 is localized at 282 the epithelial belt and its activity is required for cell-cell adhesion (Yeh et al. 2006).

283 Demonstrating that TNKS-1 is responsible for the observed PARylation is a difficult task. The 284 generation of a mammalian cell TNKS-1 knockdown has been attempted (Vyas et al. 2013), but it 285 resulted in an unviable cell line, affecting the whole cell and rapidly leading to cell death, 286 precluding a clear dissection of the underlying mechanisms. Though TNKS-2 participation 287 cannot be ruled out, it should be pointed out that its functionality as a PARylating enzyme has not 288 been proven up to date. Evaluating the detailed mechanisms involved in PAR belt synthesis as 289 well as the identification of the individual components of the E-cadherin/actin complex which are 290 PARylated, will be the subject of future research. Further work will also be necessary to analyze 291 the existence of the PAR belt in other epithelial cells, to fully characterize the biochemical 292 differences among nuclear and belt PAR, and to study the functional implications of the PAR belt 293 in different systems. 
PeerJ reviewing PDF | (v2014:06:2240:1:0:NEW 20 Sep 2014) 
295 We are indebted to MSc Pablo Liddle, technician from the Confocal Microscopy Service,

296 Facultad de Medicina, Universidad de la República, who assisted us with photography through

297 LEICA confocal microscope. We are also indebted to Gustavo Folle, Maria Vittoria Di Tomaso

298 and Ana Laura Reyes for stimulating discussions. Finally, we are grateful to Santiago Mirazzo

299 and Juan Arbiza for the cell lines.

\section{Funding Statement}

301 This work was supported by Consejo Nacional de Investigaciones Científicas y Técnicas

302 (CONICET, Argentina); Universidad de Buenos Aires (Argentina), Agencia Nacional de

303 Promoción Científica y Tecnológica (Argentina), Fundación Florencio Fiorini (Argentina), and

304 Programa de Desarrollo de las Ciencias Básicas (PEDECIBA, Uruguay).

305 S.H.F.V. is member of the Scientific Investigator Career of CONICET, Argentina. S.C.V.L is a

306 Fundación Bunge y Born post doctoral fellow. L.L.H. is member of the Sistema Nacional de

307 Investigadores of the Agencia Nacional de Investigación e Innovación (SNI, ANII, Uruguay).

\section{References}

Bonicalzi ME, Haince JF, Droit A, and Poirier GG. 2005. Regulation of poly(ADP-ribose) metabolism by poly(ADP-ribose) glycohydrolase: where and when? Cell Mol Life Sci 62:739-750.

Bonicalzi ME, Vodenicharov M, Coulombe M, Gagne JP, and Poirier GG. 2003. Alteration of poly(ADP-ribose) glycohydrolase nucleocytoplasmic shuttling characteristics upon cleavage by apoptotic proteases. Biol Cell 95:635-644.

Bottone MG, Santin G, Soldani C, Veneroni P, Scovassi AI, and Alpini C. 2012. Intracellular distribution of Tankyrases as detected by multicolor immunofluorescence techniques. Eur J Histochem 56:e4.

Burkle A, and Virag L. 2013. Poly(ADP-ribose): PARadigms and PARadoxes. Mol Aspects Med 34:1046-1065.

Cerboni B, Di Stefano A, Micheli V, Morozzi G, Pompucci G, and Sestini S. 2010. PARP activity and NAD concentration in PMC from patients affected by systemic sclerosis and lupus erythematosus. Nucleosides Nucleotides Nucleic Acids 29:471-475.

Chi NW, and Lodish HF. 2000. Tankyrase is a golgi-associated mitogen-activated protein kinase substrate that interacts with IRAP in GLUT4 vesicles. $J$ Biol Chem 275:38437-38444.

Dantzer F, and Santoro R. 2013. The expanding role of PARPs in the establishment and maintenance of heterochromatin. Febs $J$ 280:3508-3518. 
327

328

329

330

331

332

333

334

335

336

337

338

339

340

341

342

343

344

345

346

347

348

349

350

351

352

353

354

355

356

357

358

359

360

361

362

363

364

365

366

367

368

369

370

371

372

373

Fahrer J, Kranaster R, Altmeyer M, Marx A, and Burkle A. 2007. Quantitative analysis of the binding affinity of poly(ADP-ribose) to specific binding proteins as a function of chain length. Nucleic Acids Res 35:e143.

Faral-Tello P, Mirazo S, Dutra C, Perez A, Geis-Asteggiante L, Frabasile S, Koncke E, Davyt D, Cavallaro L, Heinzen H, and Arbiza J. 2012. Cytotoxic, virucidal, and antiviral activity of South American plant and algae extracts. ScientificWorldJournal 2012:174837.

Gagne JP, Isabelle M, Lo KS, Bourassa S, Hendzel MJ, Dawson VL, Dawson TM, and Poirier GG. 2008. Proteome-wide identification of poly(ADP-ribose) binding proteins and poly(ADP-ribose)-associated protein complexes. Nucleic Acids Res 36:6959-6976.

Gagne JP, Pic E, Isabelle M, Krietsch J, Ethier C, Paquet E, Kelly I, Boutin M, Moon KM, Foster LJ, and Poirier GG. 2012. Quantitative proteomics profiling of the poly(ADP-ribose)related response to genotoxic stress. Nucleic Acids Res 40:7788-7805.

Hassa PO, and Hottiger MO. 2008. The diverse biological roles of mammalian PARPS, a small but powerful family of poly-ADP-ribose polymerases. Front Biosci 13:3046-3082.

Hottiger MO, Hassa PO, Luscher B, Schuler H, and Koch-Nolte F. 2010. Toward a unified nomenclature for mammalian ADP-ribosyltransferases. Trends Biochem Sci 35:208-219.

Hsiao SJ, and Smith S. 2008. Tankyrase function at telomeres, spindle poles, and beyond. Biochimie 90:83-92.

Kawamitsu H, Hoshino H, Okada H, Miwa M, Momoi H, and Sugimura T. 1984. Monoclonal antibodies to poly(adenosine diphosphate ribose) recognize different structures. Biochemistry 23:3771-3777.

Lafon-Hughes L, Di Tomaso MV, Mendez-Acuna L, and Martinez-Lopez W. 2008. Chromatinremodelling mechanisms in cancer. Mutat Res 658:191-214.

Lehtio L, Chi NW, and Krauss S. 2013. Tankyrases as drug targets. Febs J 280:3576-3593.

Maddugoda MP, Crampton MS, Shewan AM, and Yap AS. 2007. Myosin VI and vinculin cooperate during the morphogenesis of cadherin cell cell contacts in mammalian epithelial cells. J Cell Biol 178:529-540.

Masutani M, Nakagama H, and Sugimura T. 2005. Poly(ADP-ribosyl)ation in relation to cancer and autoimmune disease. Cell Mol Life Sci 62:769-783.

Meyer-Ficca ML, Meyer RG, Coyle DL, Jacobson EL, and Jacobson MK. 2004. Human poly(ADP-ribose) glycohydrolase is expressed in alternative splice variants yielding isoforms that localize to different cell compartments. Exp Cell Res 297:521-532.

Morrison AR, Moss J, Stevens LA, Evans JE, Farrell C, Merithew E, Lambright DG, Greiner DL, Mordes JP, Rossini AA, and Bortell R. 2006. ART2, a T cell surface mono-ADPribosyltransferase, generates extracellular poly(ADP-ribose). $J$ Biol Chem 281:3336333372.

Narwal M, Venkannagari H, and Lehtio L. 2012. Structural basis of selective inhibition of human tankyrases. J Med Chem 55:1360-1367.

Ohashi S, Kanai M, Hanai S, Uchiumi F, Maruta H, Tanuma S, and Miwa M. 2003. Subcellular localization of poly(ADP-ribose) glycohydrolase in mammalian cells. Biochem Biophys Res Commun 307:915-921.

Riffell JL, Lord CJ, Ashworth A. 2012. Tankyrase-targeted therapeutics: expanding opportunities in the PARP family Nature Reviews 11: 923-936.

Strosznajder JB, Czapski GA, Adamczyk A, and Strosznajder RP. 2012. Poly(ADP-ribose) polymerase-1 in amyloid beta toxicity and Alzheimer's disease. Mol Neurobiol 46:78-84.

Tulin A, and Spradling A. 2003. Chromatin loosening by poly(ADP)-ribose polymerase (PARP) at Drosophila puff loci. Science 299:560-562.

PeerJ reviewing PDF | (v2014:06:2240:1:0:NEW 20 Sep 2014) 
374 Vilchez Larrea SC, Haikarainen T, Narwal M, Schlesinger M, Venkannagari H, Flawia MM, Villamil SH, and Lehtio L. 2012. Inhibition of poly(ADP-ribose) polymerase interferes with Trypanosoma cruzi infection and proliferation of the parasite. PLoS One 7:e46063.

Vilchez Larrea SC, Schlesinger M, Kevorkian ML, Flawia MM, Alonso GD, and Fernandez Villamil SH. 2013. Host cell poly(ADP-ribose) glycohydrolase is crucial for Trypanosoma cruzi infection cycle. PLoS One 8:e67356.

Virag L. 2013. 50Years of poly(ADP-ribosyl)ation. Mol Aspects Med 34:1043-1045.

Virag L, and Szabo C. 2002. The therapeutic potential of poly(ADP-ribose) polymerase inhibitors. Pharmacol Rev 54:375-429.

Vodenicharov MD, Ghodgaonkar MM, Halappanavar SS, Shah RG and Shah GM.2005. Mechanism of early biphasic activation of poly(ADP-ribose) polymearase-1 in response to ultraviolet B radiation. J Cell Sci118:589-599

Vyas S, Chesarone-Cataldo M, Todorova T, Huang YH, and Chang P. 2013. A systematic analysis of the PARP protein family identifies new functions critical for cell physiology. Nat Commun 4:2240.

Wahlberg E, Karlberg T, Kouznetsova E, Markova N, Macchiarulo A, Thorsell AG, Pol E, Frostell A, Ekblad T, Oncu D, Kull B, Robertson GM, Pellicciari R, Schuler H, and Weigelt J. 2012. Family-wide chemical profiling and structural analysis of PARP and tankyrase inhibitors. Nat Biotechnol 30:283-288.

Yeh TY, Meyer TN, Schwesinger C, Tsun ZY, Lee RM, and Chi NW. 2006. Tankyrase recruitment to the lateral membrane in polarized epithelial cells: regulation by cell-cell contact and protein poly(ADP-ribosyl)ation. Biochem J 399:415-425. 
397 Figure 1. PAR pools detection with different anti-PAR antibodies. PAR (green). Under 398 control conditions, Tulip clone H10 anti-PAR antibody, known to target long ramified PAR, 399 displayed no signal (data not shown). Nuclear PAR was detected both with (A) BD rabbit anti400 PAR antibody (\#551813) and (B) Tulip chicken anti-PAR antibody (\#1023). Peripheral PAR was 401 detected only with BD anti-PAR (A) suggesting differential structures of PAR polymer pools. 402 Bar: 10microm

403 Figure 2. PARP inhibitors diminished PAR belt synthesis (A-O). Vero cells were fixed $5 \mathrm{~h}$ 404 after seeding in the presence of the indicated drugs. (A-E) actin (red), (F-J) PAR (green), (K-O) 405 merge. (A, F, K) control, (B, G, L) 0.5percent dimethyl sulfoxide (DMSO; vehicle control), (C, $406 \mathrm{H}, \mathrm{M}) 5 \mathrm{mM}$ 3-AB, (D, I, N) 80 microM PJ34, (E, J, O) 5 mM 3-AB + 80 microM PJ34. Bar: 25 407 microm.

408 Figure 3. PAR vs. actin in cell-cell adhesions. (A-F) Overview; XY confocal slices (A) actin 409 microfilaments (red), (B) PAR (green), (C) merge + DAPI. Strong PAR signal delineated cell-cell 410 adhesion membrane domains whereas no signal was observed in colony borders. Bar: 10 microm. 411 (D) actin microfilaments (red), (E) PAR (green), (F) merge + DAPI. In immature cell joints, each 412 cell carried its own PAR pool. Thus, two parallel PAR lines were visible. Once membranes 413 joined, a single PAR contour was evident. Bar: 10 microm. (G-R) Orthogonal views (XY, XZ, YZ) 414 of a z stack of two neighbor cells. Yellow lines indicate cutting levels. Two main cells and the 415 border of other two cells are visible. (G, M, L, R) XY (z-projection), (H, I, N, O) XZ plane, (J, K, 416 P, Q) YZ plane. (M, N, J, P) actin (red), (I, K, L, O, Q, R) PAR (green), (H) merge, (G) merge + 417 DAPI. Arrows: PAR; double arrows: parallel PARylated cell membranes in an immature cell 418 junction; arrowheads: absent PAR in membranes lacking neighbor cells. Bar: 5 microm 
419 Figure 4. PAR and vinculin colocalization in the adhesion belts. Orthogonal views (XY, $X Z$

420 and YZ) of a z-stack. (A) XY view (z-projection). PAR (green)+ vinculin (red) + DAPI (blue). (B)

421 XZ view. (C) YZ view without DAPI; (D) XY view. PAR (green). Arrows: PAR + vinculin in the

422 PAR belt; arrowheads: non-PARylated vinculin in cell-matrix junctions. Bar: 5 microm.

423 Figure 5. Cytochalasin D induced PAR delocalization together with actin depolymerization.

424 (A-D) control, (E-H) 2 microM cytochalasin, (D, I, L) 20 microM cytochalasin D. (A, E, I) actin

425 (red), (B, F, J) PAR (green), (C, G, K) DAPI (blue), (D, H, L): merge. Arrows: PAR coexisting

426 with actin; arrowheads: PAR belt absence where actin is absent. Bar: 10 microm

427 Figure 6. EGTA and XAV 939 affected the actin cytoskeleton, cell shape and cell adhesion.

428 (A-C) Actin (red), (D-F) PAR (green), (G-I) merge. (A, D, G) control, (B, E, H) 3 mM EGTA, (C,

429 F, I) 25 microM XAV 939. Bar: 25 microm.

430 TABLE LEGENDS

431 Table 1.- Potency of PARP inhibitors towards PARP-1 and TNKSs. Binding capacity

432 expressed as $\Delta \operatorname{Tm}\left({ }^{\circ} \mathrm{C}\right)$ according to Wahlberg et al. 2012, in vitro IC50 (correspondent citations

433 in the right-most column). 3-AB: 3-aminobenzamide; OLA: Olaparib; PJ34; XAV: XAV 939.

434 While OLA is considered a selective PARP-1 inhibitor, (which does also target PARP-2, -3; and

435 -4; Narwal et al. 2012), PJ34 is a moderate potency inhibitor, displaying PARP-1 preference

436 over TNKS-1, with a lower magnitude order and XAV-939 is relatively selective for TNKS-1

437 and-2, affecting their activity 169 times more than PARP-1 activity. The inhibitory concentrations

438 in cell culture are always higher than in vitro. For example, 3-AB IC50 in vitro is $5 \mu \mathrm{M}$ and in

439 vivo, it is commonly used at a $5 \mathrm{mM}$ concentration.

440 SUPPLEMENTAL MATERIAL-FIGURE LEGENDS

441 Supplemental Figure S1. PAR belt detection in trichloroacetic acid (TCA) - or 4percent PFA

442 -fixed cells. Merged DAPI (blue) and PAR (green) channels. (A,B) TCA fixation in the absence

443 (A) or presence (B) of the primary antibody. (C, D) 4percent PFA fixation in the absence (C) or

444 presence (D) of the primary antibody. All the photographs were taken on the same confocal

445 session under the same conditions and were equally processed. PAR belt signal is clear in both

446 cases, but the background is lower with 4percent PFA. 
447 Supplemental Figure S2. PAR belt is intracellular. (A-C) PAR (green), (D-F) merged PAR

448 (green), actin (red) and DAPI (blue). (A, D) Control (usual protocol), (B, E) same protocol except

449 for the absence of permeabilization, (C, F) control with permeabilization without primary

450 antibody. Bar: $10 \mu \mathrm{m}$

451 Supplemental Figure S3. Olaparib depleted nuclear PAR without affecting peripheral PAR

452 in Vero cells. PAR (green). (A) control, (B) Olaparib (250 nM, 6 days). Bar: 20 microm. 


\section{Figure 1}

PAR pools detection with different anti-PAR antibodies.

PAR (green). Under control conditions, Tulip clone H10 anti-PAR antibody, known to target long ramified PAR, displayed no signal (data not shown). Nuclear PAR was detected both with (A) BD rabbit anti-PAR antibody (\#551813) and (B) Tulip chicken anti-PAR antibody (\#1023).

Peripheral PAR was detected only with BD anti-PAR (A) suggesting differential structures of PAR polymer pools. Bar: $10 \mu \mathrm{m}$
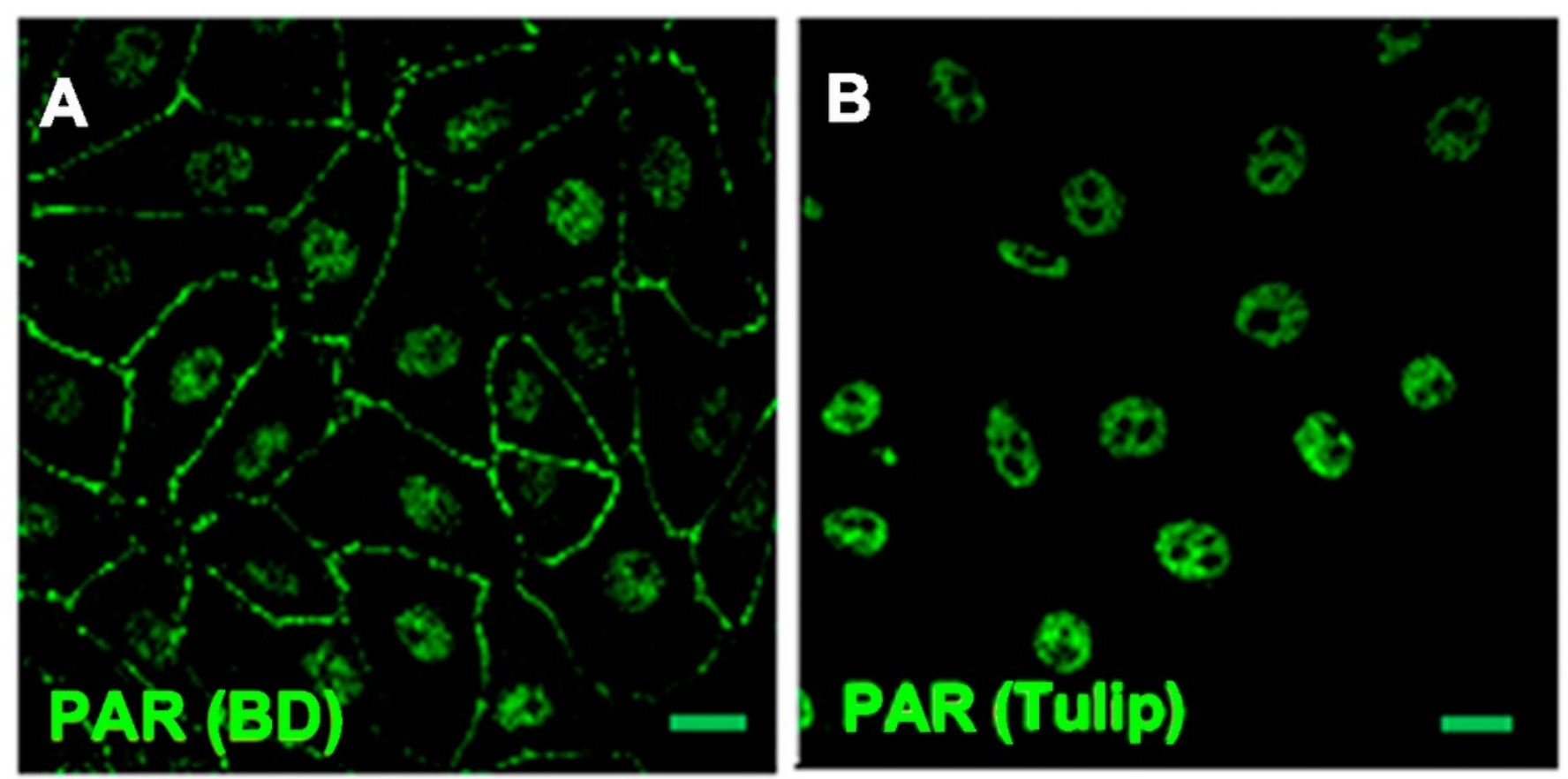


\section{Figure 2}

PARP inhibitors diminished PAR belt synthesis

(A-O). Vero cells were fixed $5 \mathrm{~h}$ after seeding in the presence of the indicated drugs. (A-E) actin (red), (F-J) PAR (green), (K-O) merge. (A, F, K) control, (B, G, L) 0.5percent dimethyl sulfoxide (DMSO; vehicle control), (C, H, M) 5 mM 3-AB, (D, I, N) 80 microM PJ34, (E, J, O) 5 mM 3-AB + 80 microM PJ34. Bar: 25 microm. 
PeerJ Reviewing Manuscript

ACTIN
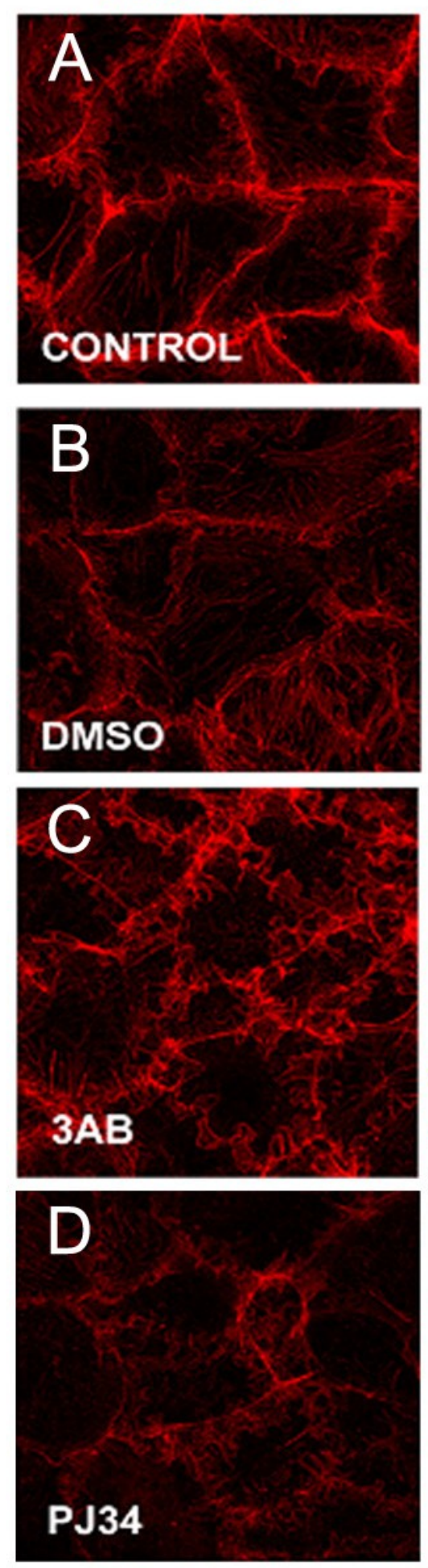

E

\section{PJ34+3AB}

PAR
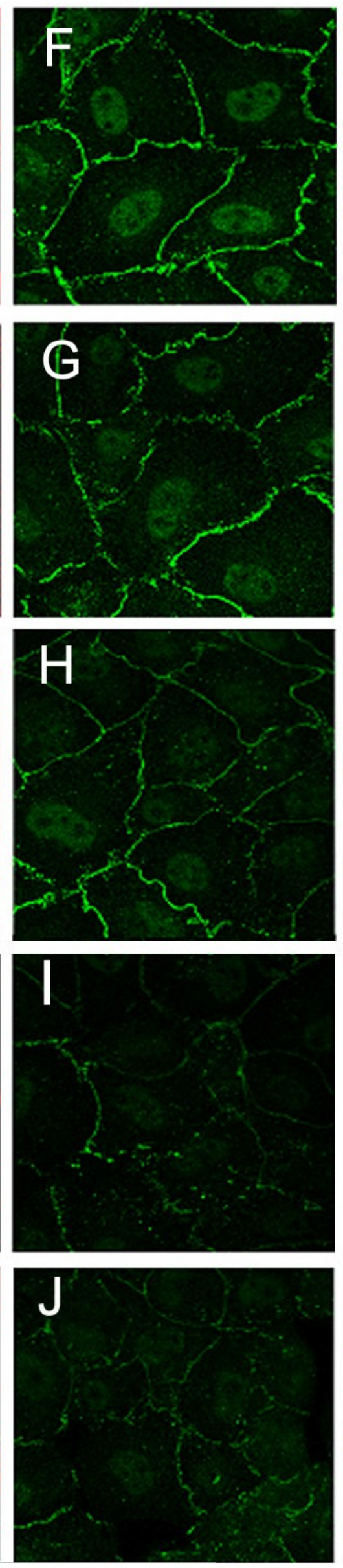

merge
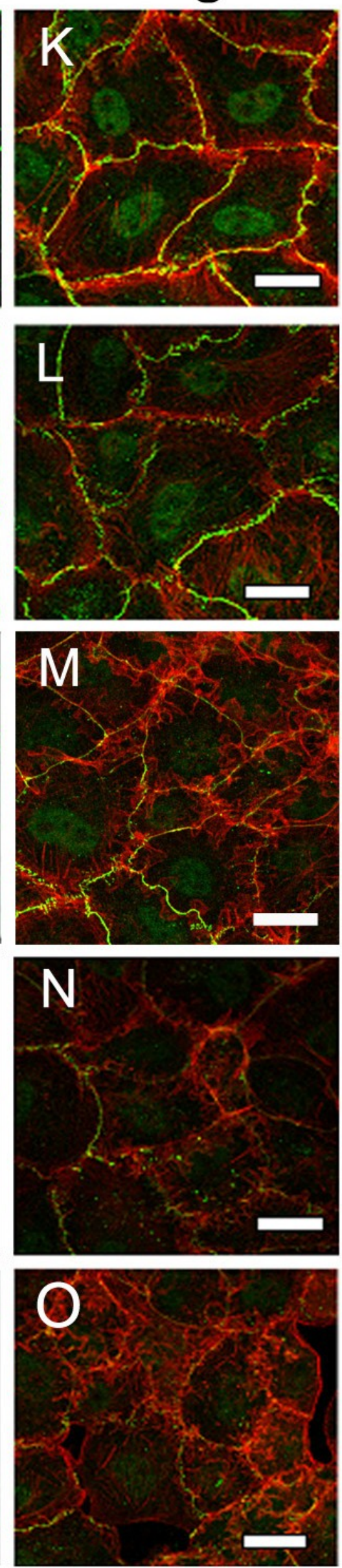


\section{Figure 3}

PAR vs. actin in cell-cell adhesions.

(A-F) Overview; XY confocal slices (A) actin microfilaments (red), (B) PAR (green), (C) merge + DAPI. Strong PAR signal delineated cell-cell adhesion membrane domains whereas no signal was observed in colony borders. Bar: 10 microm. (D) actin microfilaments (red), (E) PAR (green), (F) merge + DAPI. In immature cell joints, each cell carried its own PAR pool. Thus, two parallel PAR lines were visible. Once membranes joined, a single PAR contour was evident. Bar: 10 microm. (G-R) Orthogonal views (XY, XZ, YZ) of a z stack of two neighbor cells. Yellow lines indicate cutting levels. Two main cells and the border of other two cells are visible. (G, M, L, R) XY (z-projection), (H, I, N, O) XZ plane, (J, K, P, Q) YZ plane. (M, N, J, P) actin (red), (I, K, L, O, Q, R) PAR (green), (H) merge, (G) merge + DAPI. Arrows: PAR; double arrows: parallel PARylated cell membranes in an immature cell junction; arrowheads: absent PAR in membranes lacking neighbor cells. Bar: 5 microm 
PeerJ Reviewing Manuscript

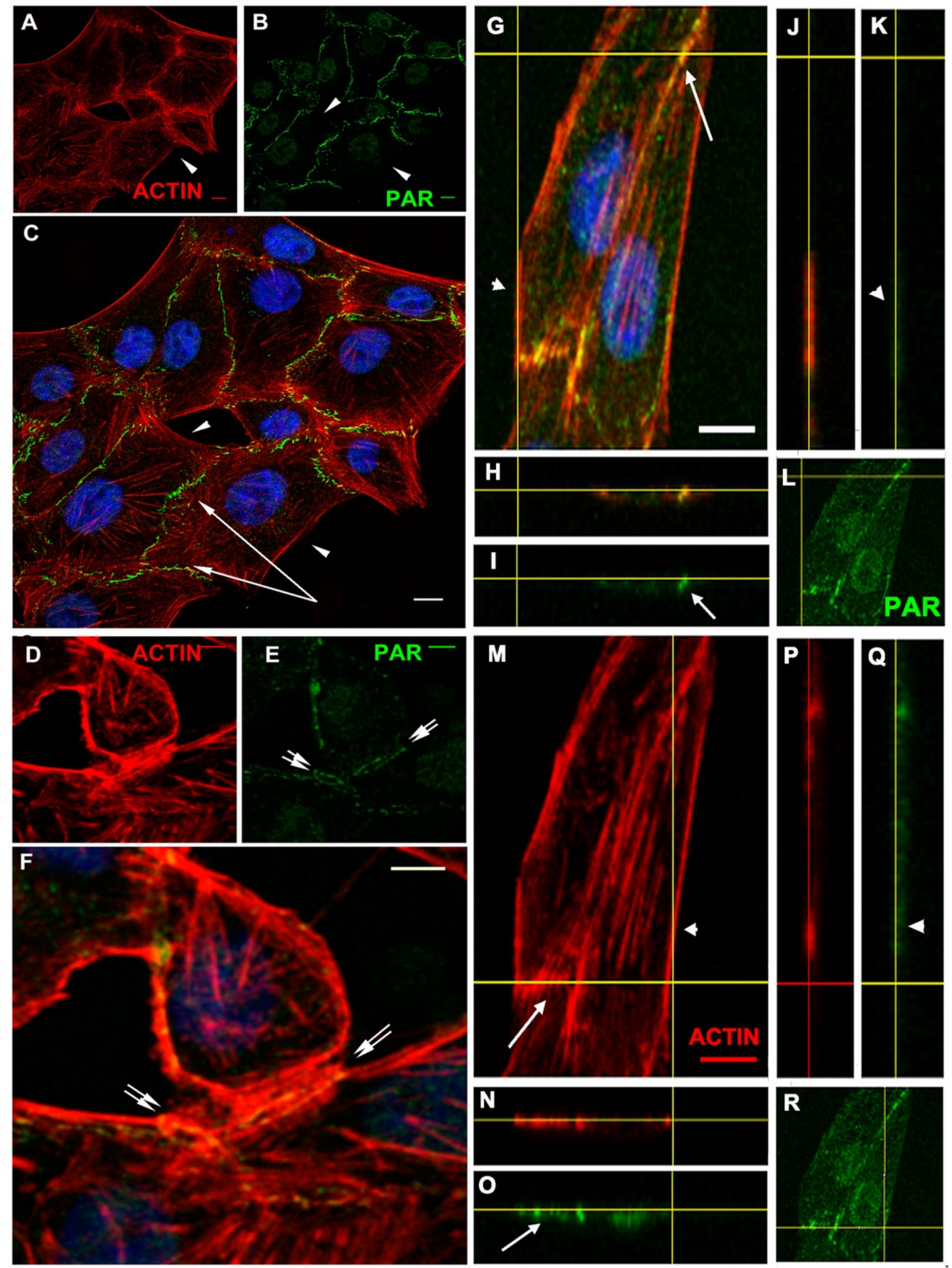




\section{Figure 4}

PAR and vinculin colocalization in the adhesion belts.

Orthogonal views ( $X Y, X Z$ and $Y Z)$ of a $Z$-stack. (A) $X Y$ view (z-projection). PAR (green)+ vinculin (red) + DAPI (blue). (B) XZ view. (C) YZ view without DAPI; (D) XY view. PAR (green). Arrows: PAR + vinculin in the PAR belt; arrowheads: non-PARylated vinculin in cellmatrix junctions. Bar: $5 \mu \mathrm{m}$.
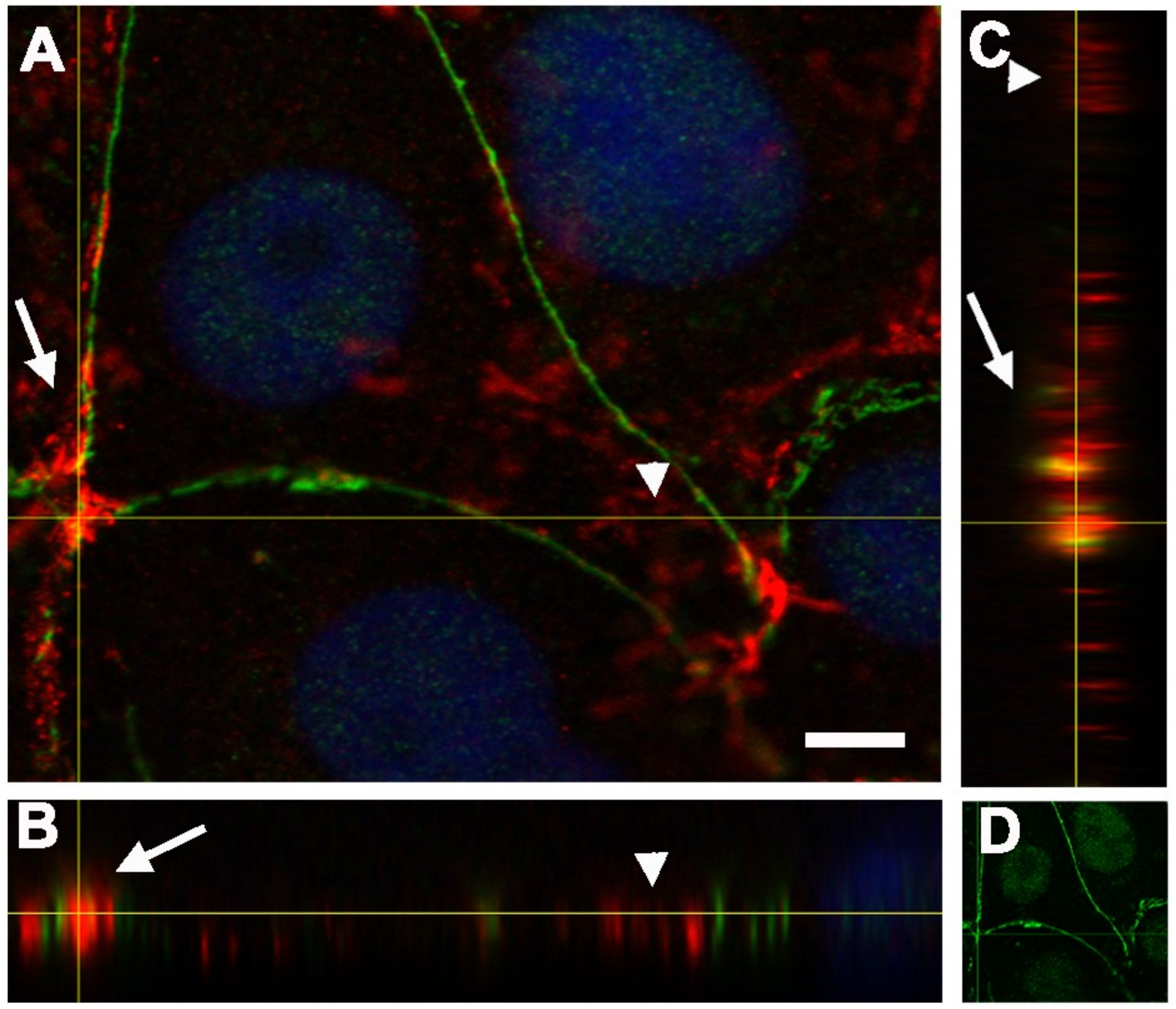


\section{Figure 5}

Cytochalasin D induced PAR delocalization together with actin depolymerization.

(A-D) control, (E-H) $2 \mu \mathrm{M}$ cytochalasin, (D, I, L) $20 \mu \mathrm{M}$ cytochalasin D. (A, E, I) actin (red), (B, F, J) PAR (green), (C, G, K) DAPI (blue), (D, H, L): merge. Arrows: PAR coexisting with actin; arrowheads: PAR belt absence where actin is absent. Bar: $10 \mu \mathrm{m}$
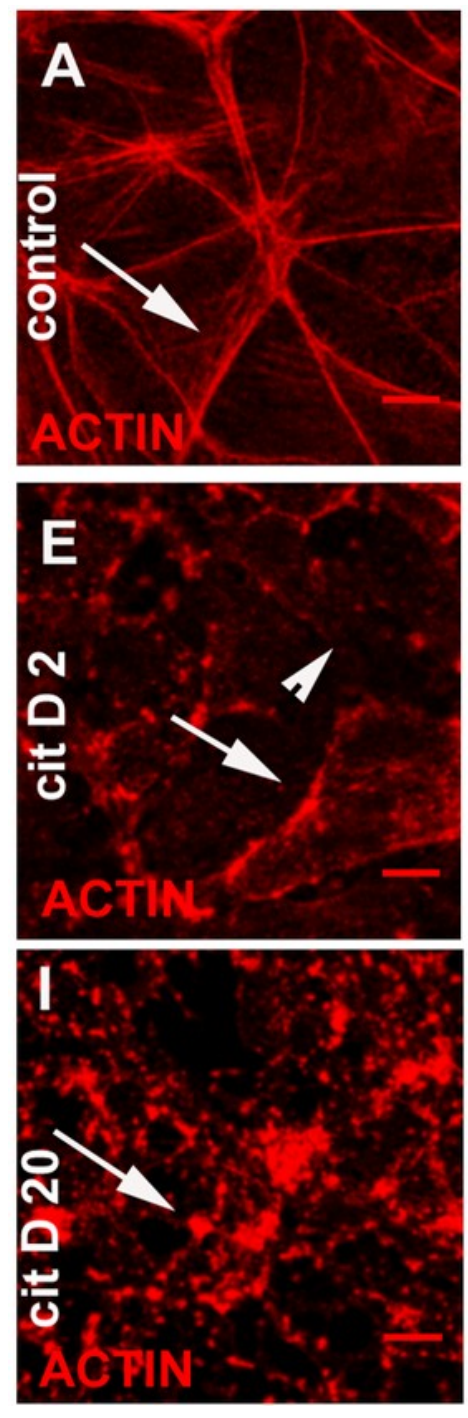
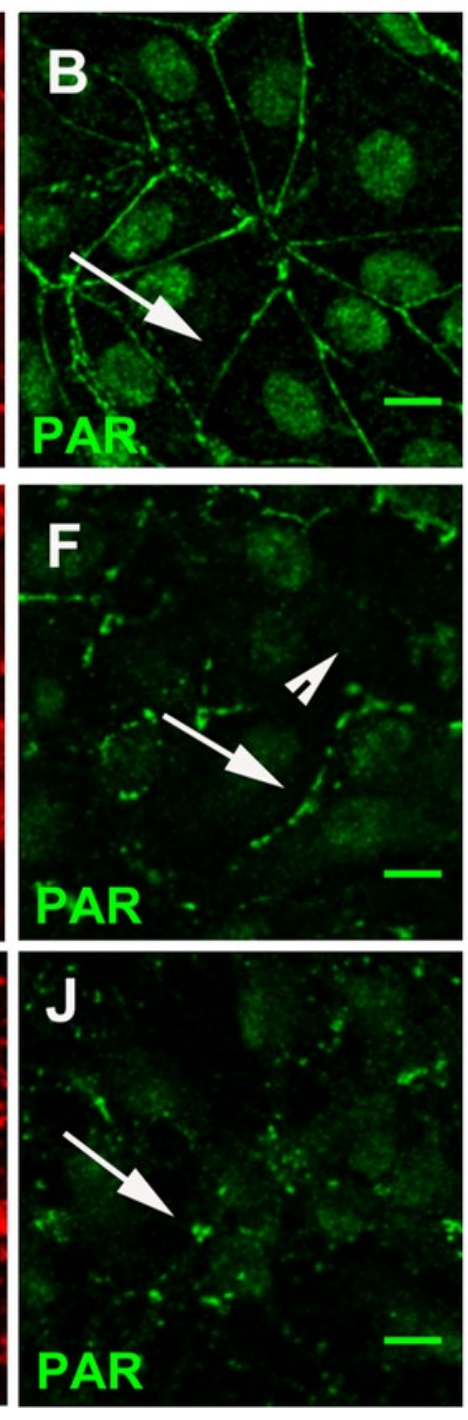
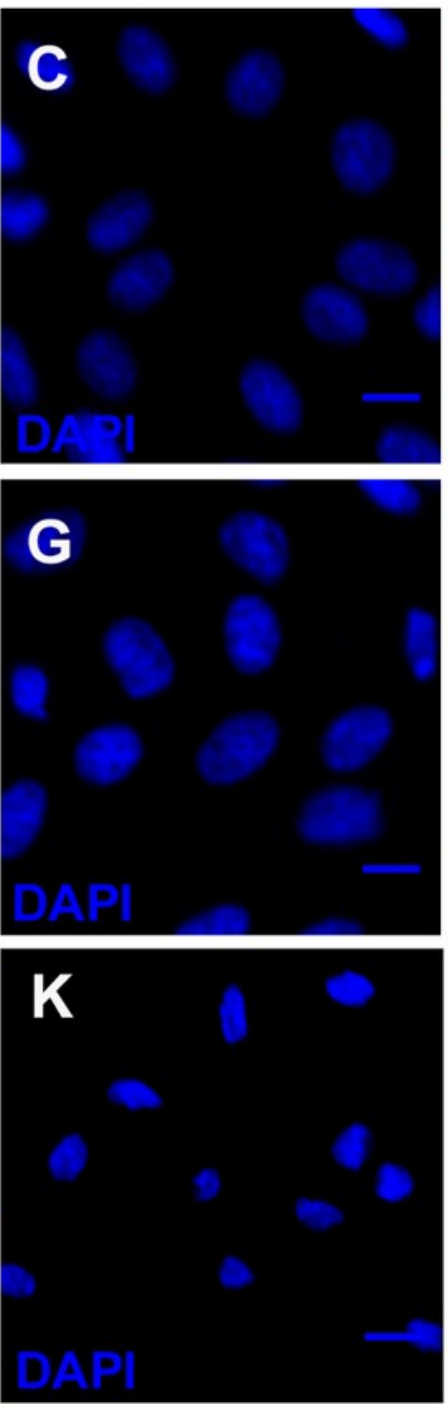
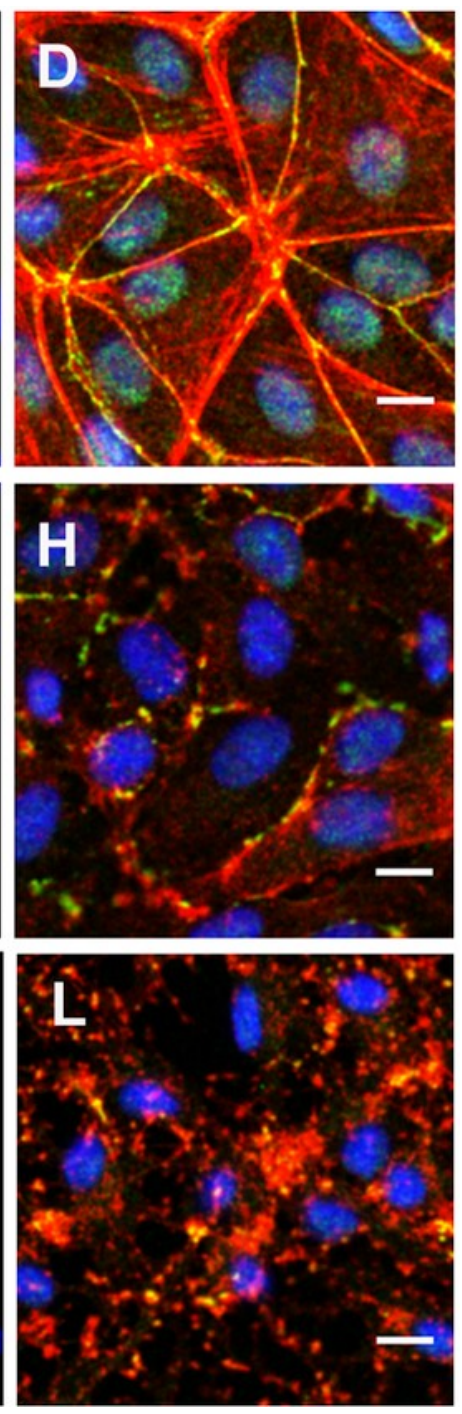


\section{Figure 6}

EGTA and XAV 939 affected the actin cytoskeleton, cell shape and cell adhesion.

(A-C) Actin (red), (D-F) PAR (green), (G-I) merge. (A, D, G) control, (B, E, H) 3 mM EGTA, (C, F, I) $25 \mu \mathrm{M}$ XAV 939. Bar: $25 \mu \mathrm{m}$. 
PeerJ Reviewing Manuscript

\section{ACTIN}
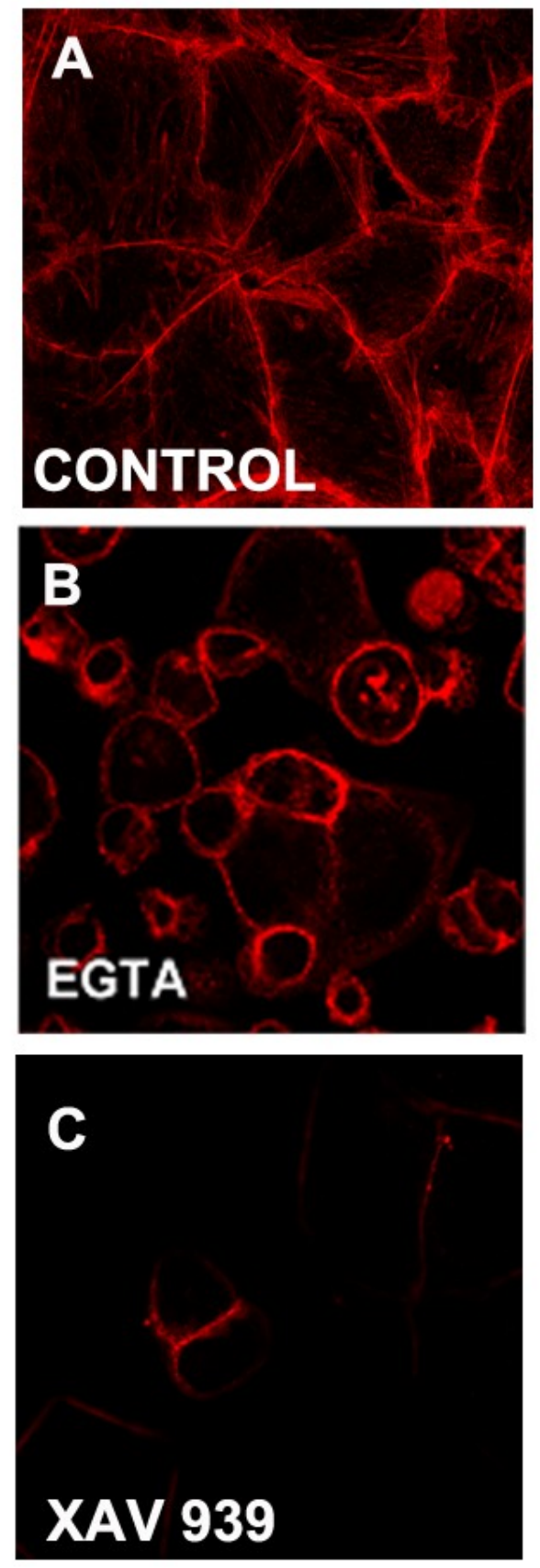

PAR
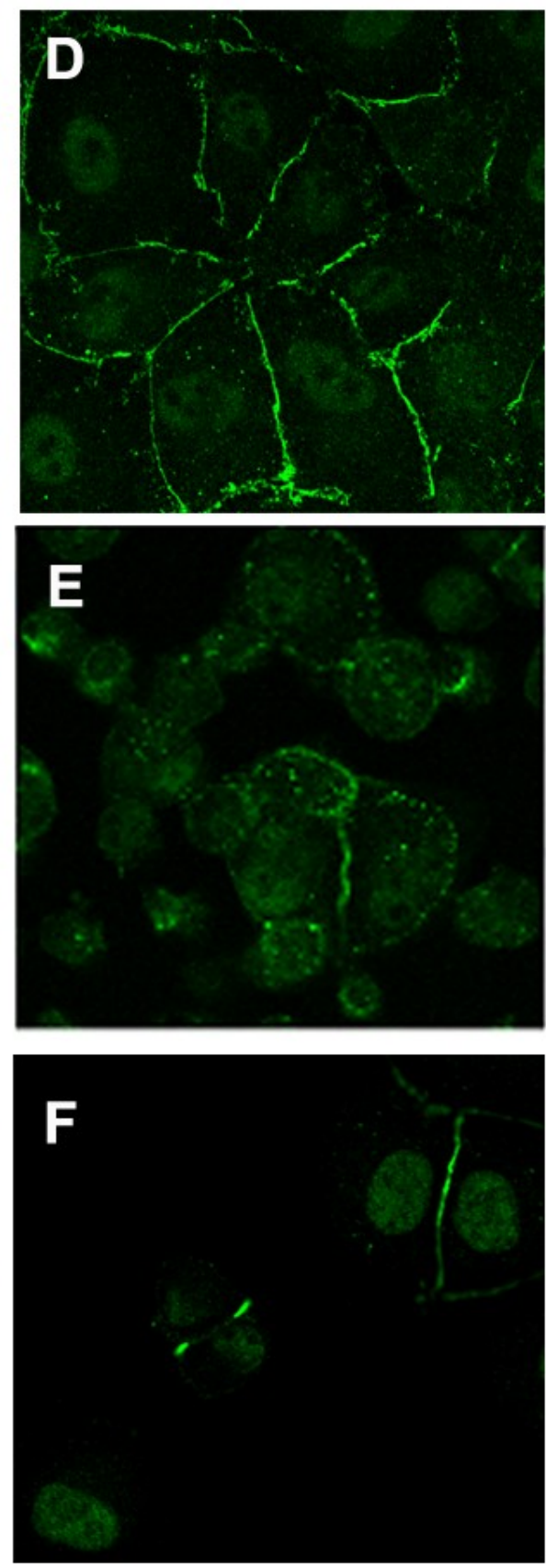

\section{merge}
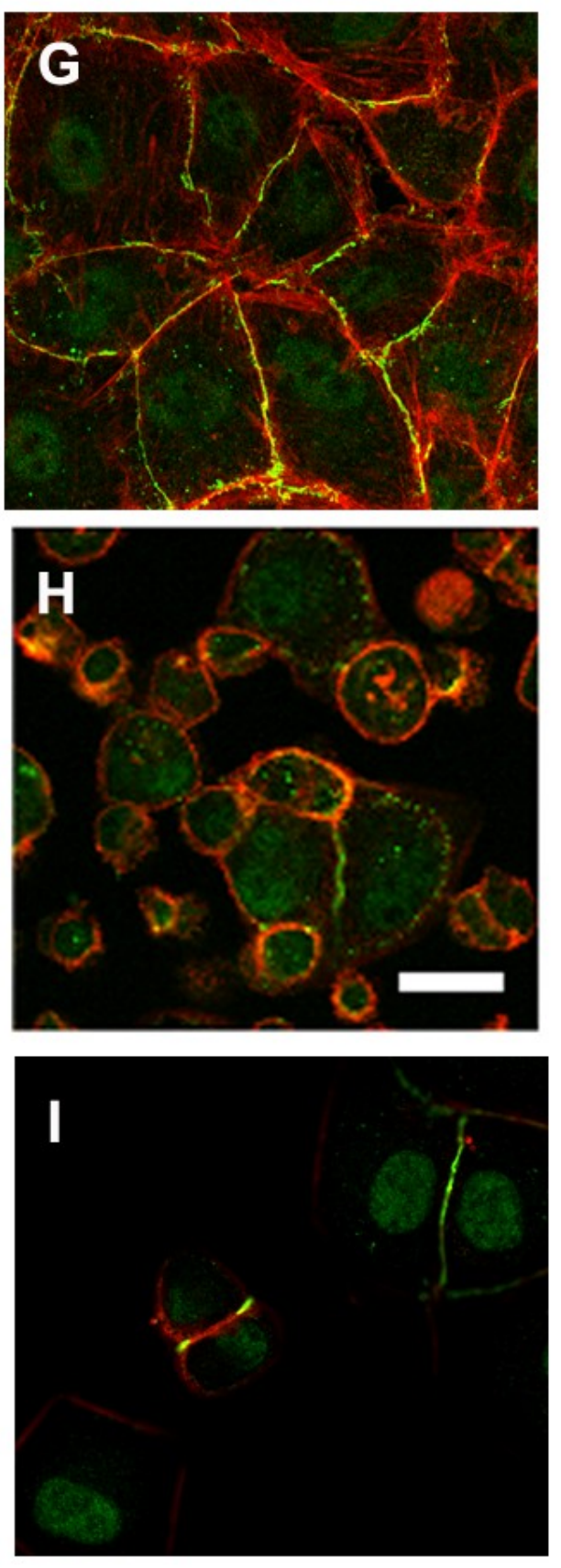


\section{Table 1 (on next page)}

Potency of PARP inhibitors towards PARP-1 and TNKSs.

Binding capacity expressed as $\Delta \operatorname{Tm}\left({ }^{\circ} \mathrm{C}\right)$ according to Wahlberg et al. 2012, in vitro IC50 (correspondent citations in the right-most column). 3-AB: 3-aminobenzamide; OLA: Olaparib;

PJ34; XAV: XAV 939. While OLA is considered a selective PARP-1 inhibitor, (which does also target PARP-2 , -3; and -4; Narwal et al. 2012) , PJ34 is a moderate potency inhibitor, displaying PARP-1 preference over TNKS-1, with a lower magnitude order and XAV-939 is relatively selective for TNKS-1 and-2, affecting their activity 169 times more than PARP-1 activity. The inhibitory concentrations in cell culture are always higher than in vitro. For example, 3-AB IC50 in vitro is $5 \mu \mathrm{M}$ and in vivo, it is commonly used at a $5 \mathrm{mM}$ concentration. 
PeerJ Reviewing Manuscript

\begin{tabular}{|c|c|c|c|c|c|c|c|}
\hline & \multicolumn{3}{|c|}{$\Delta$ Tm (oC). Interval. } & \multicolumn{3}{|c|}{ In vitro IC50 ( $\mu \mathrm{M})$} & \multirow[t]{2}{*}{ Citation } \\
\hline & hPARP-1 & $\begin{array}{c}\text { TNKS- } \\
1 \\
\end{array}$ & $\begin{array}{c}\text { TNKS- } \\
2 \\
\end{array}$ & hPARP-1 & $\begin{array}{c}\text { TNKS- } \\
1 \\
\end{array}$ & $\begin{array}{c}\text { TNKS- } \\
2 \\
\end{array}$ & \\
\hline 3AB & 1 to 3.99 & $<0.99$ & $<0.99$ & 5.400 & & & $\begin{array}{c}\text { Vilchez et al. } \\
2012\end{array}$ \\
\hline OLA & $>10$ & $<0.99$ & $<0.99$ & 0.005 & 1.500 & & $\begin{array}{c}\text { Vilchez et al. } \\
\text { 2012, Riffell et al. } \\
2012\end{array}$ \\
\hline PJ34 & 7 to 9.99 & $\begin{array}{l}1 \text { to } \\
3.99\end{array}$ & $\begin{array}{l}1 \text { to } \\
3.99\end{array}$ & 0.019 & 0.570 & - & Lehtio et al. 2013 \\
\hline XAV & 1 to 3.99 & $>10$ & $\begin{array}{l}7 \text { to } \\
9.99\end{array}$ & 2.200 & 0.013 & 0.005 & $\begin{array}{c}\text { Lehtio et al. } \\
2013, \\
\text { Riffell et al. } 2012\end{array}$ \\
\hline
\end{tabular}

Table 1. Potency of PARP inhibitors towards PARP-1 and TNKSs. 